\title{
Avaliação do oferecimento a distância de laboratório de eletrônica digital por meio de objetivos de aprendizagem e métricas do AVA
}

DOI: 10.37702/2175-957X.COBENGE.2021.3566

Reginaldo Arakaki - reginaldo.arakaki@poli.usp.br

USP

RUA SOLDADO JOAO DE OLIVEIRA 369

05140-000 - SÃO PAULO - SP

Victor Takashi Hayashi - victor.hayashi@usp.br

USP

Avenida Queiros Filho 277

09110-260 - Santo André - SP

Felipe Valencia de Almeida - felipe.valencia.almeida@usp.br

Universidade de São Pualo

Rua Marret 97

07095-140 - Guarulhos - SP

Paulo Sergio Cugnasca - paulo.cugnasca@poli.usp.br USP

Av. Prof. Luciano Gualberto, travessa 3158

05508-010 - Sao Paulo - SP

Edson Toshimi Midorikawa - edson.midorikawa@poli.usp.br USP

Avenida Professor Luciano Gualberto, travessa 3158

05508-970 - São Paulo - SP

Resumo: Este artigo descreve a avaliação do oferecimento de uma disciplina de laboratório de eletrônica digital durante o primeiro quadrimestre de 2021, com execução de atividades pré-laboratório, durante o laboratório e pós-laboratório por meio de uma solução de laboratório remoto. A disciplina foi analisada sob a perspectiva de objetivos de aprendizagem, e o monitoramento das atividades foi realizado com a coleta de métricas de submissão no Ambiente Virtual de 
Aprendizagem (AVA) Moodle. O desenvolvimento de soft skills e aprendizagem ativa por meio de uma abordagem de Project Based Learning (PBL) foram oportunidades identificadas neste relato de experiência.

Palavras-chave: Educação a distância (EAD), Avaliação de ensino, Laboratório digital, Project Based Learning (PBL), soft skills 


\section{Avaliação do oferecimento a distância de laboratório de eletrônica digital por meio de objetivos de aprendizagem e métricas do AVA}

\section{INTRODUÇÃO}

O objetivo pedagógico da disciplina PCS3635 Laboratório Digital I da Escola Politécnica da USP (Figura 1) é o estabelecimento de métodos para construir sistemas digitais simples, utilizando componentes de pequena complexidade, como contadores, memória, e operações simples que caracterizam uma Unidade Lógica Aritmética como adição e comparação.

Figura 1 - Objetivos da Disciplina

"Desenvolvimento de projetos com circuitos digitais simples.

Observação prática e montagem com dispositivos de

eletrônica digital (FPGA e componentes discretos).

Treinamento em depuração. Familiarização com

instrumentação de bancada. Treinamento de trabalhos em

grupo. Aprendizado dos processos de documentação de

circuitos digitais."

Fonte: (JUPITERWEB, 2021)

No contexto do oferecimento da disciplina no ano de 2021, as aulas foram divididas em duas partes. Inicialmente foram planejadas sete experiências para a primeira metade da disciplina, que são apresentadas na Tabela 1. A função dessas experiências é familiarizar o aluno tanto com o aparato experimental quanto com conceitos técnicos que serão aplicados intensivamente no laboratório. Nessa primeira metade da disciplina é dado um foco especial nas placas Field Programmable Gate Array (FPGA) e na linguagem de descrição de hardware VHDL. Já na segunda metade da disciplina os alunos dedicam-se à concepção e ao desenvolvimento de um projeto com tema livre durante as quatro semanas seguintes.

Tabela 1 - Atividades planejadas no ambiente virtual de aprendizagem.

\begin{tabular}{|c|c|}
\hline Experiência & Título \\
\hline 1 & Primeiro Contato com Circuitos Digitais em VHDL \\
\hline 2 & Um Fluxo de Dados Simples \\
\hline 3 & Desenvolvendo um Fluxo de Dados \\
\hline 4 & Familiarização com Internet das Coisas (loT) \\
\hline 5 & Considerações de Projeto de Circuitos em FPGA \\
\hline 6 & Projeto Base do Jogo do Desafio da Memória \\
\hline 7 & Projeto do Jogo do Desafio da Memória \\
\hline & Fonte: Autoria própria
\end{tabular}

Alguns pontos adicionais foram inseridos na disciplina correspondentes à formação do aluno como um profissional da Engenharia de Computação. São algumas habilidades bastante apreciadas pela indústria e que se destacam nas atividades das aulas. São elas: 
- Criatividade: Neste caso, os grupos de alunos foram desafiados a criarem uma variação de jogo do "Genius", utilizado como ponto de partida para o projeto final da disciplina, explorando aspectos como jogabilidade, controle, complexidade de níveis, painel de resultados entre outros. Neste caso, a criatividade é estimulada, desenvolvida e avaliada nos requisitos de projeto;

- Resiliência: O fato de se criar uma ideia não garante uma pontuação no projeto final da disciplina. O projeto deve ser robusto e preciso, conforme os requisitos estabelecidos. E então, outra habilidade é desenvolvida e amadurecida, a resiliência: o projeto deve funcionar. Para isso, a solução, os testes e os ajustes devem ser organizados para que os erros de projeto sejam sistematicamente detectados, corrigidos num ciclo virtuoso de conceber, projetar e testar por partes. O aluno constata a importância de saber avançar por partes, recuar nos pontos mais complexos para dividir o problema em partes menores, para fazer funcionar cada parte e então avançar para o objetivo final;

- Colaboração em time: $O$ aluno entende que cada integrante do grupo tem as suas diferenças de atitude, de resistência, de paciência, e até mesmo de conhecimento. Como lidar com essas diferenças e fazer com que cada esforço contribua para o resultado é um desafio. A colaboração ocorre pela interação, por meio das cobranças mútuas, pelo compartilhamento de conhecimento e experiências, considerando que as orientações dos professores devam ser aplicadas em prol das metas de projeto. Este é um aspecto do aprendizado que ocorre de maneira bastante produtiva;

- Gestão do tempo: É muito comum que os alunos se empolguem com o projeto e a divisão de tempo seja desequilibrada. Para isso, eles têm que desenvolver a habilidade de como organizar os tempos com as outras atividades de outras disciplinas. Um aspecto importante na disciplina é que os alunos percebem rapidamente que as 3 horas e 40 minutos de uma aula de laboratório podem parecer muito tempo, mas isso é muito relativo. Se as atividades não forem organizadas e previamente planejadas, todo o tempo de laboratório se esvai rapidamente em uma série de tentativas e erros, de maneira improdutiva;

- Gestão de complexidade: Os professores orientam os alunos a lidarem com a complexidade da seguinte forma. Qual a complexidade do projeto criado? Os recursos e conhecimento disponíveis serão suficientes? Algum requisito deve ser simplificado? Qual o caminho da implementação que ajuda a avaliar a viabilidade dos requisitos estabelecidos? Esse aprendizado é destacado nos requisitos não funcionais, muitas vezes extraídos da criatividade do grupo. Os alunos aprendem a recuar ou a simplificar alguns requisitos, e com isso, são treinados a equilibrar o tempo e recursos disponíveis para a implementação e entregas do projeto;

- Gestão de comunicação: As técnicas ágeis de projeto, muito em uso na indústria, incluem a gestão do projeto. Os alunos participam de reuniões similares ao Scrum Daily Meetings, onde o plano de entrega por Sprint, a identificação dos riscos das atividades e como mitigá-los são treinados nas aulas de projeto. Para isso, toda a turma, cada um no seu grupo, aprendem como se comunicar de maneira direta e eficaz antes, durante e após a sessão de aula em laboratório.

Uma solução de laboratório remoto (ALMEIDA, 2021) foi utilizada na transformação da disciplina do presencial para o oferecimento remoto, de forma a viabilizar a execução de experimentos de forma remota com uso de mecanismos de acesso remoto, controle e monitoramento de sinais analógicos e digitais pelo computador, e monitoramento dos resultados por meio de webcam. Esta solução foi utilizada no oferecimento de outra disciplina em 2020 (HAYASHI, 2020). 
Em relação aos trabalhos anteriores, este trabalho se diferencia por apresentar um relato de experiência de uma perspectiva pedagógica e não apenas técnica.

\section{$2 \quad$ A DISCIPLINA SOB A PERSPECTIVA PEDAGÓGICA}

Uma versão revisada da taxonomia de Bloom (KRATHWOHL, 2002) é comumente utilizada para classificar objetivos educacionais, de forma a facilitar a comunicação daquilo que os educadores esperam que os alunos aprendam, por meio da definição dos objetivos de aprendizagem. Além do estabelecimento de expectativas claras para os alunos, o uso da taxonomia também auxilia na definição de métodos de avaliação mais objetivos. Esta taxonomia compreende uma hierarquia cumulativa de complexidade, considerando as seguintes categorias: conhecimento, compreensão, aplicação, análise, síntese e avaliação, conforme a Figura 2 (GALHARDI, 2013).

Figura 2 - Taxonomia de Bloom revisada

\begin{tabular}{|l|l|l|l|l|l|}
\hline 1-Lembrar & 2-Entender & 3-Aplicar & 4-Analisar & 5-Avaliar & 6-Criar \\
\hline Reconhecer & Interpretar & Executar & Diferenciar & Verificar & Gerar \\
\hline Relembrar & Exemplificar & Implementar & Organizar & Criticar & Planejar \\
\hline Listar & Classificar & Computar & Atribuir & Julgar & Produzir \\
\hline Nomear & Sumarizar & Resolver & Comparar & Recomendar & Criar \\
\hline Definir & Inferir & Demonstrar & Contrastar & Justificar & Inventar \\
\hline Escrever & Comparar & Utilizar & Separar & Apreciar & Desenvolver \\
\hline Apontar & Explicar & Construir & Categorizar & Ponderar & $\begin{array}{l}\text { Elaborar } \\
\text { hipóteses }\end{array}$ \\
\hline
\end{tabular}

Fonte: (GALHARDI, 2013)

Conforme o exposto, fica fundamentada a definição de objetivos de aprendizagem para cada aula, de forma a permitir a definição das atividades de absorção dos alunos, e métodos de avaliação destas atividades. A partir das apostilas e apresentações do Ambiente Virtual de Aprendizagem - AVA Moodle, é possível definir os seguintes objetivos de aprendizagem:

Experiência Dirigida 1 - "Primeiro Contato com Circuitos Digitais em VHDL":

- (1 - Lembrar) Relembrar síntese e implementação de circuitos digitais em VHDL e em placa de desenvolvimento com FPGA

- (2 - Entender) Interpretar um circuito digital simples

- (3 - Aplicar) Utilizar software para projeto de circuitos digitais

Experiência Dirigida 2 - "Um Fluxo de Dados Simples":

- (2 - Entender) Explicar o planejamento e atividades práticas

- (3 - Aplicar) Utilizar o laboratório remoto para síntese do circuito em uma placa FPGA

- (6 - Criar) Desenvolver um projeto hierárquico com descrição estrutural VHDL 
Experiência Dirigida 3 - "Desenvolvendo um Fluxo de Dados":

- (2 - Entender) Explicar o planejamento e atividades práticas

- (2 - Entender) Interpretar um fluxo de dados de um circuito digital

- (3 - Aplicar) Utilizar um módulo de memória

- (3 - Aplicar) Utilizar um display de 7 segmentos

- (6 - Criar) Desenvolver um projeto com descrição estrutural VHDL

Experiência Dirigida 4 - "Familiarização com Internet das Coisas (loT)":

- (2 - Entender) Explicar o planejamento e atividades práticas

- (3 - Aplicar) Utilizar um módulo loT ESP8266 do laboratório

- (3 - Aplicar) Demonstrar o controle de leds da FPGA usando celular

- (6 - Criar) Criar um projeto em um aplicativo

Experiência Dirigida 5 - "Considerações de Projeto de Circuitos em FPGA":

- (2 - Entender) Interpretar a interface de circuitos digitais com elementos externos de entrada de dados

- (3 - Aplicar) Utilizar o laboratório remoto para síntese do circuito em uma placa FPGA

- (6 - Criar) Desenvolver um projeto de circuito digital com sinal periódico como clock

Experiência Dirigida 6 - "Projeto Base do Jogo do Desafio da Memória":

- (2 - Entender) Interpretar o código de componentes

- (6 - Criar) Produzir o projeto de fluxo de dados e de unidade de controle de circuitos digitais

- (6 - Criar) Desenvolver um projeto de circuito digital a partir de sua especificação

Experiência Dirigida 7 - "Projeto do Jogo do Desafio da Memória":

- (2 - Entender) Interpretar a interface com elementos externos e plataformas loT

- (3 - Aplicar) Utilizar o laboratório remoto para síntese do circuito em uma placa FPGA

- (6 - Criar) Desenvolver um projeto de circuito digital a partir de sua especificação

\section{Projeto:}

- (3 - Aplicar) Utilizar o laboratório remoto para síntese do circuito em uma placa FPGA

- (6 - Criar) Inventar uma aplicação inovadora para o projeto

- (6 - Criar) Planejar um projeto de circuito digital

- (6 - Criar) Desenvolver um projeto de circuito digital a partir de sua especificação

Uma alternativa à aprendizagem passiva é a aprendizagem ativa, que prevê uma postura mais ativa por parte do aluno, que pode atuar na resolução de problemas e no desenvolvimento de projetos. Esta última possibilidade é suportada pela abordagem de aprendizagem baseada em projetos, ou Project Based Learning - PBL (VALENTE, 2013).

Considerando como motivação as oportunidades do PBL, a segunda parte do curso consistiu na especificação, desenvolvimento e apresentação de um projeto de um jogo a partir dos módulos que os alunos tiveram contato nos experimentos dirigidos na primeira parte do curso. Este conjunto de atividades fomentou o desenvolvimento das soft skills definidas na seção anterior, conforme ilustrado na Figura 3. 
Figura 3 - Desenvolvimento das soft skills durante as atividades da disciplina

\begin{tabular}{|c|c|c|c|c|c|c|}
\hline \multirow{2}{*}{ Soft Skill } & \multicolumn{3}{|c|}{ Experimentos Dirigidos } & \multicolumn{3}{c|}{ Projeto } \\
\cline { 2 - 7 } & Pré-Aula & Aula & Pós-Aula & Pré-Aula & Aula & Pós-Aula \\
\hline Criatividade & & $\checkmark$ & & $\checkmark$ & & \\
\hline Resiliência & $\checkmark$ & $\checkmark$ & $\checkmark$ & $\checkmark$ & $\checkmark$ & $\checkmark$ \\
\hline Colaboração & $\checkmark$ & $\checkmark$ & & $\checkmark$ & \\
\hline $\begin{array}{c}\text { Gestão de } \\
\text { Tempo }\end{array}$ & & $\checkmark$ & & $\checkmark$ & $\checkmark$ & \\
\hline $\begin{array}{c}\text { Gestão de } \\
\text { Complexidade }\end{array}$ & & $\checkmark$ & & $\checkmark$ & $\checkmark$ & \\
\hline \begin{tabular}{c} 
Comunicação \\
\hline
\end{tabular} & & $\checkmark$ & & $\checkmark$ & \\
\hline
\end{tabular}

Fonte: Autoria própria

\section{MÉTODO DE COLETA DE MÉTRICAS NO AVA}

A Figura 4 ilustra o método utilizado para a coleta de métricas de submissões no Moodle. No Ambiente Virtual de Aprendizagem - AVA é possível obter duas planilhas: a planilha de participantes e seus respectivos perfis, e a planilha com o histórico de notas e submissões. Estas duas planilhas suportam o levantamento das atividades realizadas pelos alunos, a partir da exclusão dos dados dos perfis de monitor e professor. As atividades previstas podem ser obtidas com a observação das atividades previstas para cada aula. A comparação entre atividades previstas e realizadas pode ser mais granular se, além do aspecto temporal registrado em um gráfico de evolução das submissões (obtido a partir dos dados de atividades realizadas), as atividades forem classificadas de acordo com categorias de interesse. Para a disciplina de laboratório de eletrônica digital, duas seções foram consideradas: atividades dos experimentos dirigidos da primeira parte da disciplina, e atividades relativas a um projeto prático na segunda parte da disciplina. Adicionalmente, as atividades também foram classificadas de acordo com seu momento de realização: antes da aula, durante a aula e após a aula. 
Figura 4 - Método utilizado para a coleta de métricas no AVA

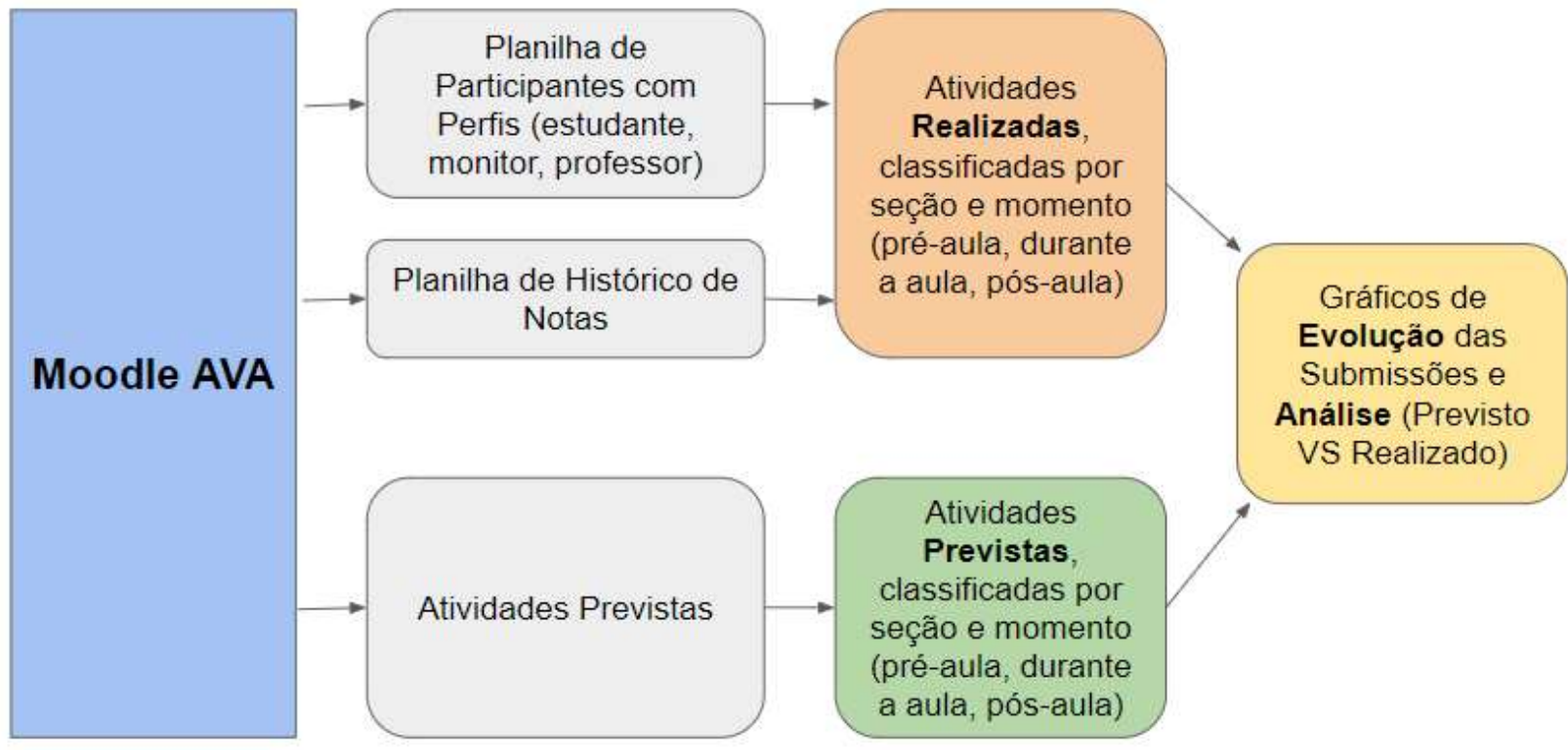

Fonte: Autoria própria

\section{RESULTADOS OBTIDOS}

O ambiente de aprendizagem virtual (AVA) Moodle foi utilizado durante o oferecimento da disciplina entre janeiro e abril de 2021, com participação de 48 alunos do curso cooperativo de Engenharia da Computação. Neste ambiente, um total de 44 atividades diferentes foram realizadas com os alunos. Estas atividades ocorreram em três diferentes momentos: antes das aulas, durante as aulas e após as aulas, conforme a Tabela 2.

Tabela 2 - Atividades planejadas no ambiente virtual de aprendizagem.

\begin{tabular}{|c|c|}
\hline Atividades do Moodle & Subtotal de Atividades \\
\hline Planejamento dos Experimentos Dirigidos(Pré-Aula) & 6 \\
Teste(Durante a Aula) & 14 \\
Prova(Durante a Aula) & 2 \\
Relatório dos Experimentos Dirigidos(Durante e Pós-Aula) & 7 \\
Especificação do Projeto(Pré-aula) & 2 \\
Planejamento das Atividades do Projeto(Pré-Aula) & 4 \\
Apresentações Intermediárias do Projeto (Durante a aula) & 3 \\
Relatórios Intermediários do Projeto (Pós-aula) & 4 \\
Resultado Final do Projeto (Pós-aula) & 2 \\
\hline Total & $\mathbf{4 4}$ \\
\hline
\end{tabular}

Fonte: Autoria própria 
Durante a primeira parte do curso, referente aos experimentos dirigidos, as seguintes atividades foram realizadas com apoio do AVA:

- Pré-aula: planejamento dos experimentos dirigidos

- Durante a aula: testes e provas

- Pós-aula: relatório técnico

De forma similar, as seguintes atividades para o projeto da segunda parte do curso foram realizadas no AVA:

- Pré-aula: especificação, planejamento das atividades do projeto

- Durante a aula: apresentações intermediárias do projeto

- Pós-aula: relatórios técnicos intermediários e final

Ao organizar as atividades nos três momentos (antes, durante e depois da aula), pode-se observar uma grande quantidade de atividades no período durante a aula na primeira parte do curso, e uma menor quantidade de atividades durante a aula na segunda parte do curso, como pode ser observado na Figura 5.

Figura 5 - Atividades no Moodle

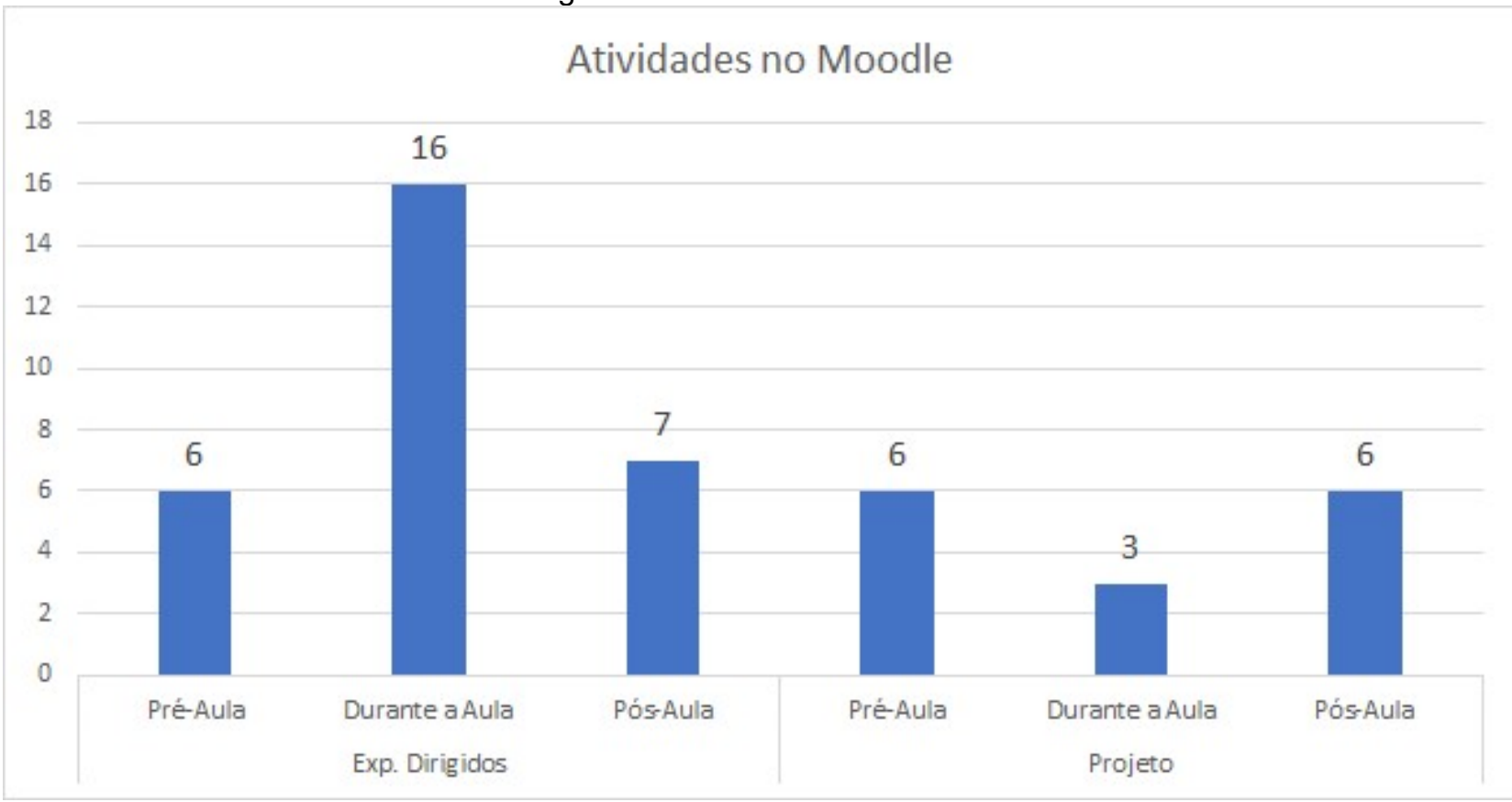

Fonte: Autoria própria

Foram realizadas um total de 2821 submissões de atividades no ambiente Moodle pelos 48 alunos (organizados em duplas durante os experimentos, exceto nas provas práticas individuais) no decorrer do curso. Conforme a Figura 6, o quantitativo destas submissões segue em linhas gerais o quantitativo das atividades da Figura 5. 
Figura 6 - Submissões no Moodle

Submissões por Tipo de Atividade

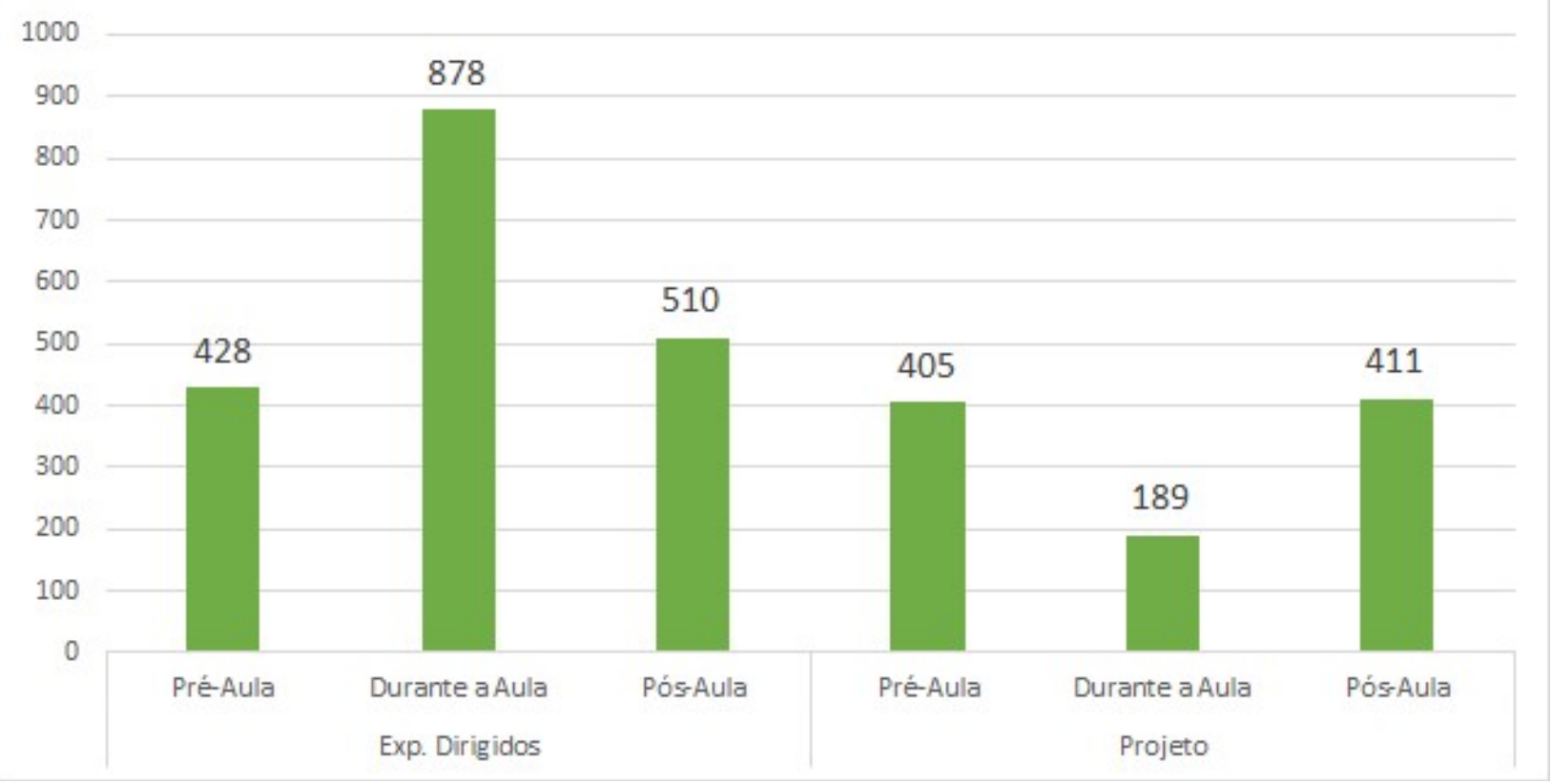

Fonte: Autoria própria

A evolução ao longo do tempo destas submissões do Moodle pode ser observada na Figura 7. Cabe destacar dois pontos: um número menor de submissões na primeira semana do curso, e na penúltima semana do curso.

Figura 7 - Evolução das Submissões no Moodle

Evolução das Submissões no Moodle

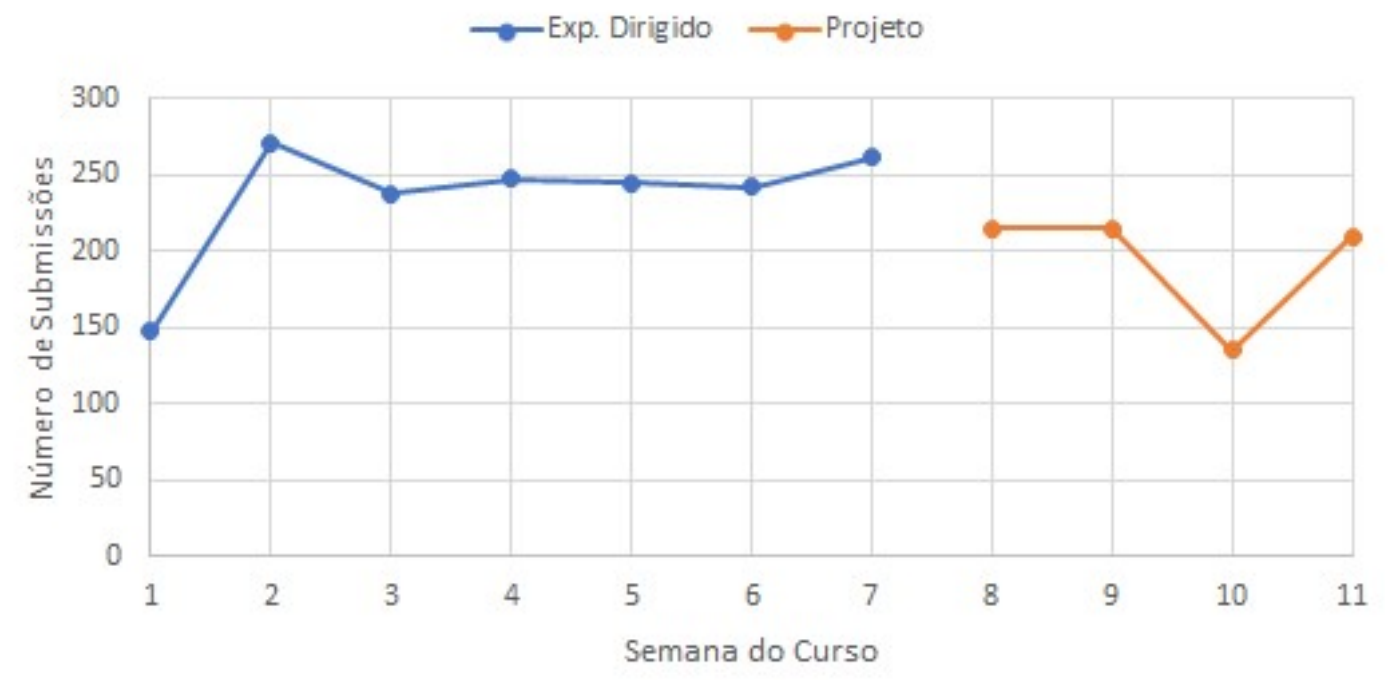

Fonte: Autoria própria 
O detalhamento na Figura 8 quanto às submissões na primeira parte do curso mostra que uma justificativa para o menor número de submissões na primeira semana foi a ausência do planejamento pré-aula por se tratar de uma aula inaugural da disciplina.

Figura 8 - Evolução das Submissões dos Experimentos Dirigidos no Moodle

\section{Evolução das Submissões - Exp. Dirigidos}

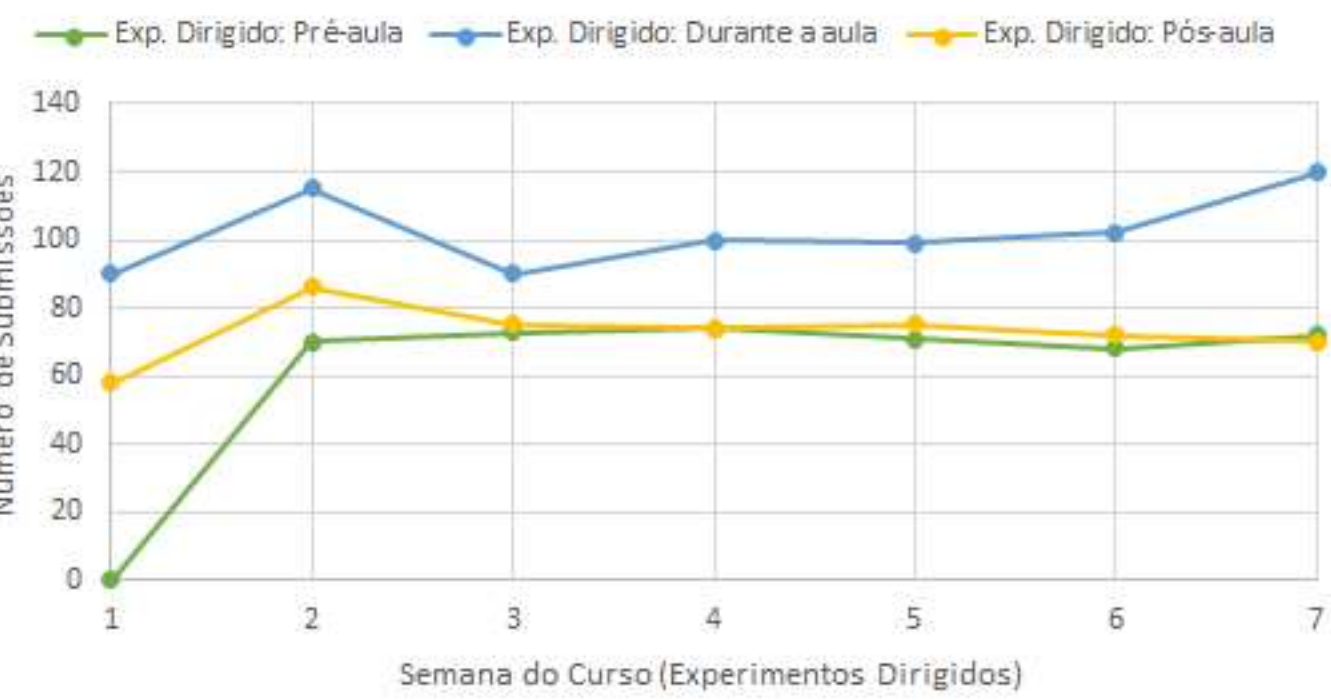

Fonte: Autoria própria

Por fim, conforme a Figura 9, observa-se que a pequena queda nas submissões na terceira semana de projeto não ocorreu em uma única etapa. Conforme aviso no Moodle, isso ocorreu devido aos feriados decretados pela Prefeitura de São Paulo entre 26 de março e 1 de abril de 2021, que levaram ao cancelamento das aulas da semana 3, e disponibilização de horários de OpenLab, em caráter opcional.

Figura 9 - Evolução das Submissões do Projeto no Moodle

\section{Evolução das Submissões - Projeto}

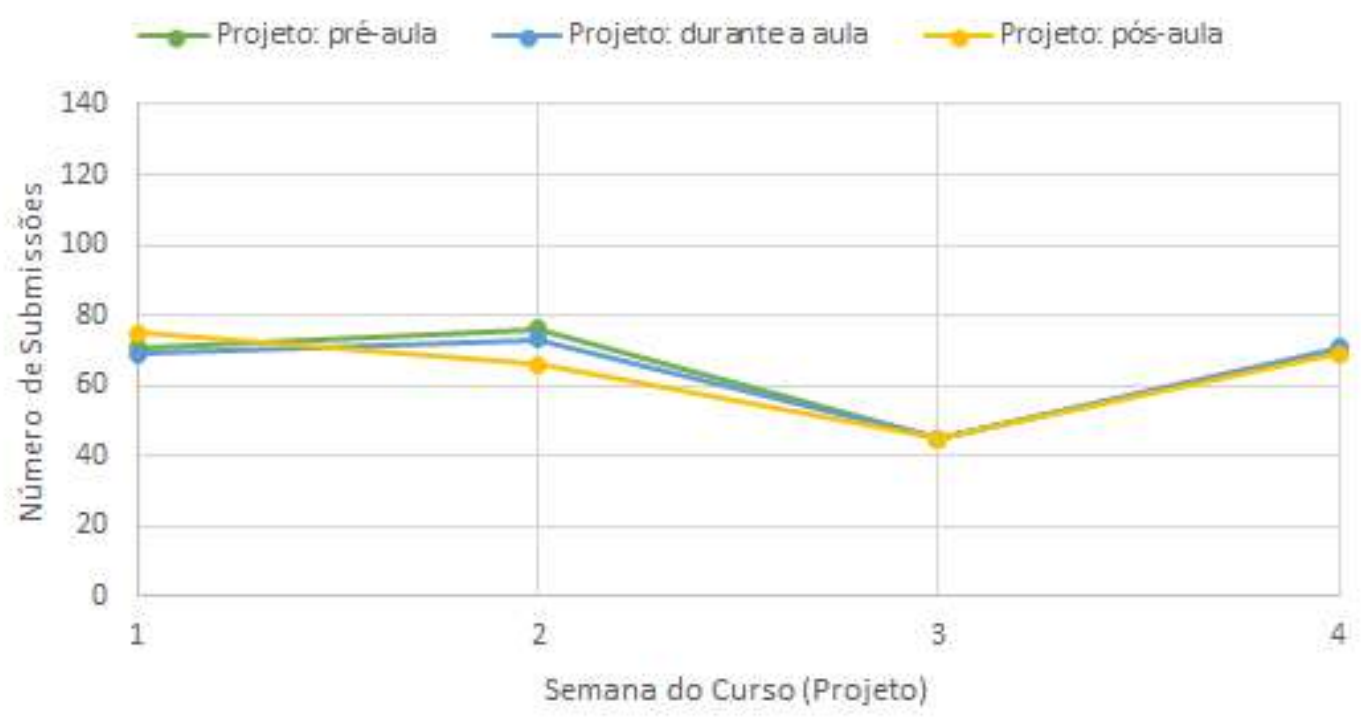

Fonte: Autoria própria 


\section{CONSIDERAÇÕES FINAIS}

Neste artigo foi apresentada a avaliação de uma disciplina de laboratório de eletrônica digital oferecida no primeiro quadrimestre de 2021 sob a perspectiva de objetivos de aprendizagem para planejamento de atividades pré-aula, durante a aula e pós-aula. Um diagnóstico por meio de métricas de submissões do Ambiente Virtual de Aprendizagem (AVA) Moodle permitiu identificar um ponto de melhoria na terceira semana de projeto.

Há que destacar que mesmo com o distanciamento social imposto pela pandemia do Covid-19, habilidades interessantes foram praticadas, como a prática de colaborar, aprendendo como respeitar a diversidade de atitudes e de métodos dos colegas para buscar os resultados dos projetos. As práticas de comunicar para os professores e demais colegas a situação do projeto, incluindo o andamento e as dificuldades foram treinadas por reuniões similares a reuniões ágeis praticadas na indústria como o Daily Scrum Meeting. A criatividade foi outra habilidade desenvolvida a partir da qual, com base em um projeto de referência, os grupos foram estimulados a pesquisarem mais sobre o jogo de memória e usando os recursos disponíveis, como criar funcionalidades interessantes aos usuários. Por fim, também foi desenvolvida a habilidade de gestão dos alunos para lidar com os recursos limitados como tempo, material e conhecimento para realizar entregas significativas ao longo do projeto.

\section{Agradecimentos}

Agradecemos ao Departamento de Computação e Sistemas Digitais (PCS) da Escola Politécnica da USP pelo apoio e a todos os alunos e os técnicos que contribuíram para o sucesso no oferecimento desta disciplina.

\section{REFERÊNCIAS}

\section{Trabalhos em eventos}

ALMEIDA, Felipe; HAYASHI, Victor; ARAKAKI, Reginaldo; MIDORIKAWA, Edson; CUGNASCA, Paulo; CANOVAS, Sergio. Laboratório Digital à Distância: Percepções de Docentes e Discentes. In: I SIMPÓSIO BRASILEIRO DE EDUCAÇÃO EM COMPUTAÇÃO (EDUCOMP), 2021. Disponível em: https://sol.sbc.org.br/index.php/educomp/article/view/14499. Acesso em 27 abr. 2021

GALHARDI, Antonio César; AZEVEDO, Marilia Macorin de. Avaliações de aprendizagem: o uso da taxonomia de Bloom. In: Anais do VII Workshop Pós-graduação e Pesquisa do Centro Paula Souza, São Paulo. 2013. p. 237-247.

HAYASHI, Victor; ALMEIDA, Felipe; ARAKAKI, Reginaldo; MIDORIKAWA, Edson; CUGNASCA, Paulo; CANOVAS, Sergio. DESAFIOS E OPORTUNIDADES PARA O ENSINO REMOTO DA DISCIPLINA DE LABORATÓRIO DE ELETRÔNICA DIGITAL. In: XLVIII Congresso Brasileiro de Educação em Engenharia, 2020. Disponível em: http://abenge.org.br/sis submetidos.php?acao=abrir\&evento=COBENGE20\&codigo=COB ENGE20 00145 00003298.pdf. Acesso em 27 abr. 2021

KRATHWOHL, David R. A revision of Bloom's taxonomy: An overview. Theory into practice, v. 41, n. 4, p. 212-218, 2002. 
VALENTE, José Armando. Aprendizagem Ativa no Ensino Superior: a proposta da sala de aula invertida. Depto. de Multimeios, Nied e GGTE-Unicamp \& Ced-PucSP, 2013.

\section{Internet:}

JUPITERWEB. Ementa da disciplina de Laboratório Digital I. Disponível em:

https://uspdigital.usp.br/jupiterweb/obterDisciplina?nomdis=\&sgldis=PCS3635. Acesso em: 26 abril 2021.

\section{EVALUATION OF THE DIGITAL ELECTRONICS LAB REMOTE OFFERING WITH LEARNING OBJECTIVES AND METRICS COLLECTED ON VLE}

Abstract: This paper describes the evaluation of the digital electronics lab remote offering during 2021 first quadrimester, with pre-lab, during lab, and post-lab activities supported by a remote lab solution. The course was analyzed from a learning objectives' perspective, and its monitoring was realized with submission metrics collected in the Moodle Virtual Learning Environment (VLE). Soft skills development and active learning with a Project Based Learning (PBL) approach were identified opportunities in this experiment report.

Keywords: Remote education, Education evaluation, Digital lab, Project Based Learning (PBL), soft skills. 